\title{
Pulsed Neutron Imaging Based Crystallographic Structure Study of a Japanese Sword made by Sukemasa in the Muromachi Period
}

\author{
Kenichi Oikawa ${ }^{1, a^{*}}$, Yoshiaki Kiyanagi ${ }^{2, b}$, Hirotaka Sato ${ }^{3, c}$, Kazuma Ohmae $^{2, d}$, \\ Anh Hoang Pham ${ }^{5,}$, Kenichi Watanabe ${ }^{2, \mathrm{f}}$, Yoshihiro Matsumoto ${ }^{4, g}$, \\ Takenao Shinohara ${ }^{1, \mathrm{~h}}$, Tetsuya Kai ${ }^{1, \mathrm{i}}$, Stefanus Harjo ${ }^{1, \mathrm{j}}$, Masato Ohnuma ${ }^{3, \mathrm{k}}$, \\ Sigekazu Morito ${ }^{5,1}$, Takuya Ohba ${ }^{5, m}$, Akira Uritani $^{2, \mathrm{n}}$, Masakazu Ito ${ }^{6,0}$ \\ ${ }^{1}$ J-PARC Center, Japan Atomic Energy Agency, Ibaraki, 319-1195, Japan \\ ${ }^{2}$ Graduate School of Engineering, Nagoya University, Aichi, 464-8603, Japan \\ ${ }^{3}$ Faculty of Engineering, Hokkaido University, Hokkaido 060-8628, Japan \\ ${ }^{4}$ Comprehensive Research Organization for Science and Society, Ibaraki 319-1106, Japan \\ ${ }^{5}$ Interdisciplinary Faculty of Science and Engineering, Shimane University, Shimane 690-8504, \\ Japan \\ ${ }^{6}$ WAKOU MUSEUM, Shimane 692-0011, Japan \\ akenichi.oikawa@j-parc.jp, 'biyanagi@phi.phys.nagoya-u.ac.jp, ch.sato@eng.hokudai.ac.jp, \\ doomae.kazuma@f.mbox.nagoya-u.ac.jp, eanhpham@riko.shimane-u.ac.jp, ${ }^{\mathrm{f}} \mathrm{k}$ - \\ watanabe@energy.nagoya-u.ac.jp, 9y_matsumoto@cross.or.jp, htakenao.shinohara@j-parc.jp, \\ 'tetsuya.kai@j-parc.jp, jstefanus.harjo@j-parc.jp, kohnuma.masato@eng.hokudai.ac.jp, \\ Imosh@riko.shimane-u.ac.jp, mohba@riko.shimane-u.ac.jp, "uritani@energy.nagoya-u.ac.jp, \\ ${ }^{\circ}$ masakazu_itoh@cup.ocn.ne.jp
}

Keywords: Japanese Sword, Quench Hardening, Martensite, Bragg-Edge Transmission, RITS Code

Abstract. Energy-resolved neutron imaging using a pulsed neutron source is capable of visualizing crystallographic information over a large area of a sample by analyzing positiondependent Bragg-edge transmission spectra. We applied this method to a Japanese sword, signed by Sukemasa, to elucidate position dependent crystallographic characteristics, including but not limited to: degree of hardening, crystallite size, degree of preferred orientation. By comparing the degree of hardening to that of a contemporary short Japanese sword (dagger), the Sukemasa showed relatively small changes in the position of Bragg-edge (110) and its broadening. No coarse grain was found within the detector resolution (ca. $1 \mathrm{~mm}$ ), and the crystallite size of the blade area was analyzed to be almost uniform and less than $1 \mu \mathrm{m}$. We thus recognize in a comprehensive manner that the Sukemasa sword was manufactured with great care.

\section{Introduction}

Japanese swords are very attractive not only as a work of art but also from a metallurgical point of view. There were several famous traditional styles (Gokaden for instance) of Japanese swordmaking in the Koto (old sword) age; A.D. 987-1596. Detailed manufacturing techniques, such as methods of obtaining raw materials [1], an iterative hammering process and the method for combining different steels [2] in the Koto age, are not clear, since they were handed down secretly within each school and have been lost over time. Various kinds of Japanese swords were sliced thickly and the cut surface were studied by conventional methods, such as microscopy and chemical analysis, EPMA, and so on [3]. This analytical approach was possible in the past, but at the present time when Japanese vintage swords have become valuable, it is indispensable to

(c) (1) Content from this work may be used under the terms of the Creative Commons Attribution 3.0 license. Any further distribution of this work must maintain attribution to the author(s) and the title of the work, journal citation and DOI. Published under license by Materials Research Forum LLC. 
establish non-destructive analysis methods to identify some peculiar characteristics related to the sword making procedure. Neutron experiments are a powerful tool to study metallic cultural heritage objects due to their high penetrating power and capability to give micro-structural properties [4,5]. Bragg-edge transmission (BET) imaging, in particular, gives real-space distributions of bulk information in the Japanese sword [6]. In this work, we investigated crystallographic information of a Japanese sword made by Sukemasa in Izumi province (southern part of Osaka prefecture; out of Gokaden) in the first quarter of the 16th century.

\section{Experimental}

The Japanese sword Sukemasa (Fig. 1(a); $790 \mathrm{~mm}$ in total length and $15 \mathrm{~mm}$ in curvature) and a contemporary dagger (300 mm blade length divided into three $100 \mathrm{~mm}$ pieces) were used in the present investigation.

The time-of-flight (TOF) neutron transmission imaging experiment was performed at BL22 RADEN [7] in MLF J-PARC with a proton beam power of $150 \mathrm{~kW}$. The downstream sample position with a pinhole optical geometry was used as shown in Fig. 1(b). Two-dimensional TOF BET spectra between 0.5 and $6.5 \AA$ were obtained by use of a gas electron multiplier (GEM) detector [8] with a $0.8 \times 0.8 \mathrm{~mm}^{2}$ pixel resolution and a $10 \times 10 \mathrm{~cm}^{2}$ detection area. Three different areas of the sword were measured, as indicated in Fig. 1(a). The BET imaging measurement time was about 8 hours for each sample position and 6 hours without the sample for the detector calibration.

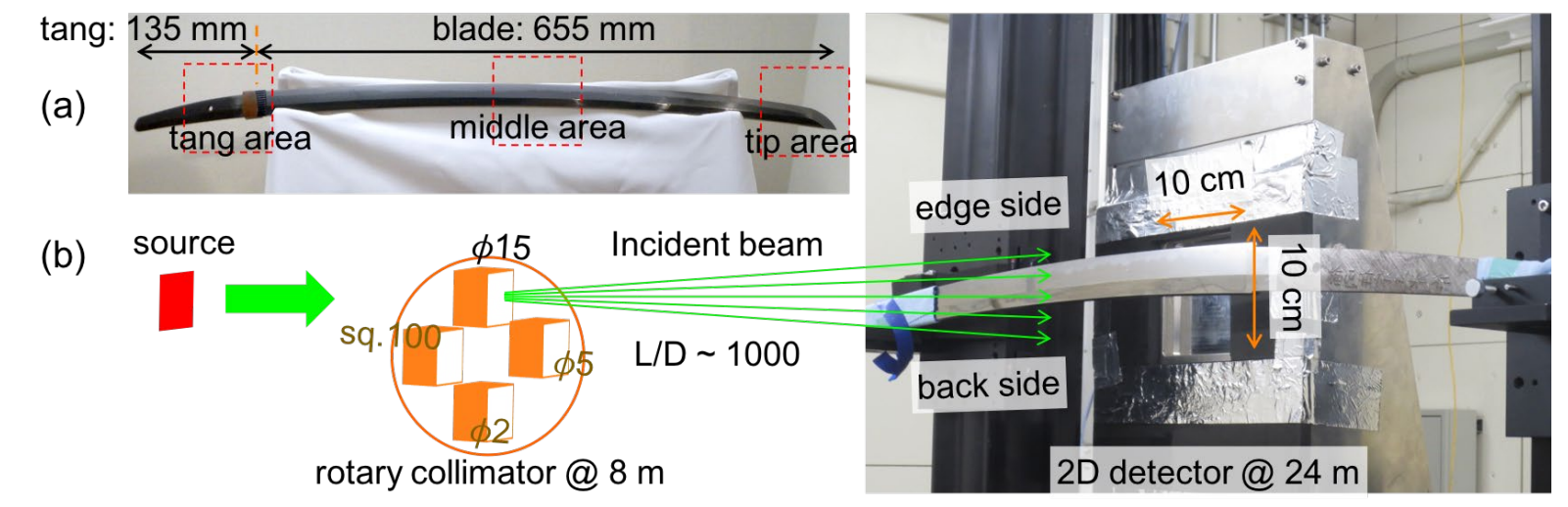

Fig. 1 (a) A picture of the Sukemasa. Three measured areas are indicated by the dashed red boxes. (b) A schematic view and a picture of the BET measurement at RADEN.

\section{Data analysis}

\section{Wavelength-range contrast imaging}

The wavelength dependent neutron transmission spectrum of a crystalline sample is expressed as follows:

$$
\operatorname{Tr}(\lambda)=\exp \left(-\sum_{p} \sigma_{\text {tot }, p}(\lambda) \rho_{p} t_{p}\right)
$$

where $\sigma_{\text {tot }, p}(\lambda)$ is the neutron total cross section, $\rho_{p}$ is the density, and $t_{p}$ is the thickness of the crystalline phase $p$. Here, we tentatively define $T r_{\text {cold }}$ and $T r_{\text {thermal }}$ where the former and latter are the wavelength

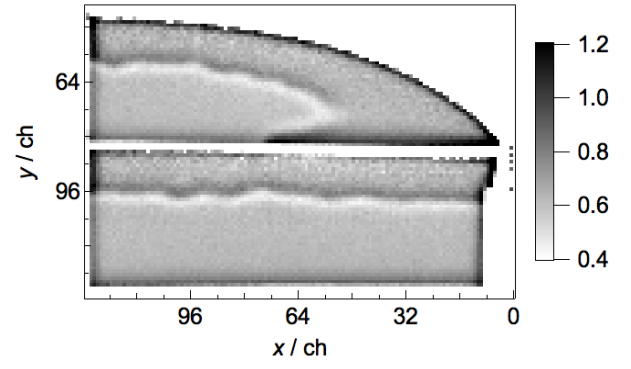

Fig. 2 The wavelength-range contrast image of the tip and middle of the contemporary dagger. 
of 4.1-6.4 and 0.5-2.3 $\AA$, respectively. A wavelength-range contrast image is obtained by the ratio, $\operatorname{Ln}\left(\operatorname{Tr}_{\text {cold }}\right) / \operatorname{Ln}\left(\operatorname{Tr}_{\text {thermal }}\right)$, where the dependence on $\rho_{p} t_{p}$ is canceled. Therefore, the image represents the division of the wavelength-range dependent $\sigma_{\text {tot, }, \text { }}$, i.e. $\sigma_{\text {cold }} / \sigma_{\text {thermal }}$.

Fig. 2 shows the wavelength-range contrast image of the contemporary dagger. Figs. 3(a), 3(b) and 3(c) show the wavelength-range contrast images of the tang, middle and tip areas of the Sukemasa, respectively. The quenching boundary of the Sukemasa shows a lower contrast than the contemporary dagger. The magnetic and/or small angle scattering could affect this particular contrast seen in the contemporary dagger, but this is still under investigation.
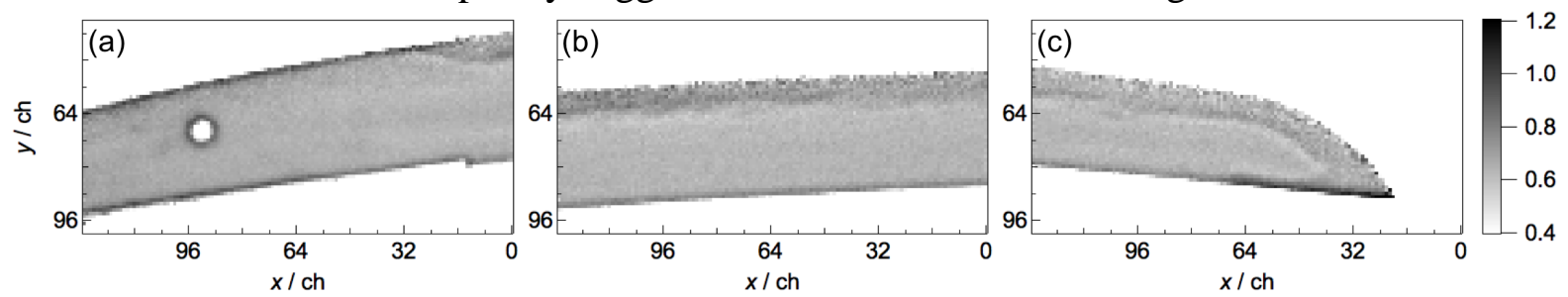

Fig. 3 The wavelength-range contrast images of (a) the tang, (b) middle and (c) tip areas of the Sukemasa. Note that a small circle in the tang area is the Mekugi (fastening pin) hole.

\section{Single edge and full pattern analysis using the RITS code}

All BET spectra were analyzed by a Rietveld-like analysis code, RITS [9]. A single-edge analysis was performed to obtain the lattice (110) plane spacing $d_{110}$ and the broadening of the edge width $w_{110}$, whereas a Rietveld-type analysis was performed to obtain the projected atomic number density, crystallite size and preferred orientation parameter. For all analyses, a singlephase body-centered-cubic structure was assumed.

Fig. 4(a) exemplifies the Rietveld-type fitting pattern for a BET spectrum where wavelengths between 2.0 and $4.5 \AA$ were used. Fig. 4(b) exemplifies the single edge fitting pattern for a (110) Bragg-edge in the BET spectrum for wavelengths between ca. 3.8 and 4.3 A. To obtain enough data statistics for the analysis, BET spectra for a $2 \times 2$ pixel $(1.6 \times 1.6$ $\mathrm{mm}^{2}$ ) area were summed into a single BET spectrum, and the pixel area was stepped at 1 pixel $(0.8 \mathrm{~mm})$ intervals in the $x$ - and $y$ directions.

\section{Result and discussion}

\section{Single-edge analysis}

One of the essential processes for the
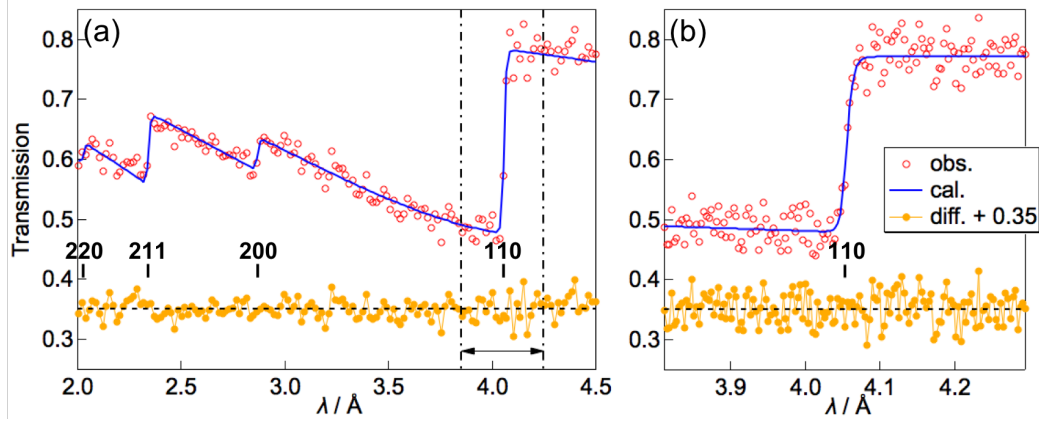

Fig. 4 Examples of (a) Rietveld-type analysis for a full BET spectrum with 100- $\mu$ s time channels and (b) single-edge analysis for the Bragg-edge (110) with 20- $\mu$ s time channels.

The vertical dashed lines in (a) indicate the range for the single-edge analysis.
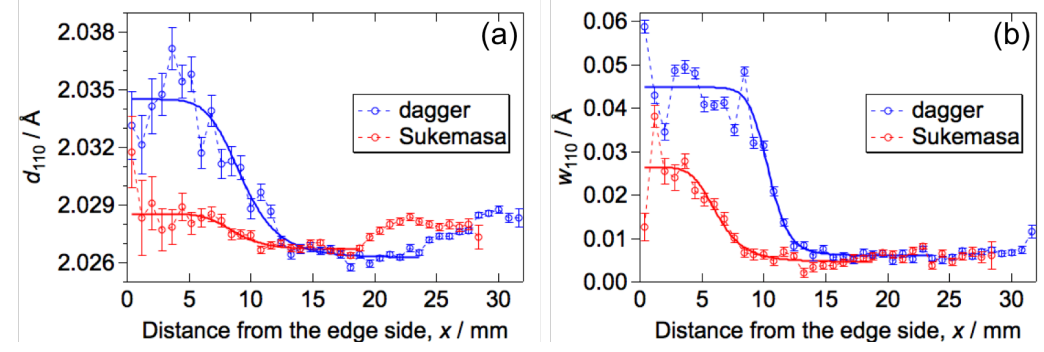

Fig. 5 Distribution of the lattice d-spacing (a) and broadening of the edge width (b) of the (110) plane across the middle area of the Sukemasa and the contemporary dagger. 
fabrication of a Japanese sword is the unique quenching method with an intentional temperature gradient, which produces a hard martensite phase and soft pearlite - ferrite phase along the cutting-edge and the back side of the blade, respectively [10]. As the quenching technique should be different in age and tradition, the martensite characteristics; such as the lattice $d$-spacing and broadening of the edge width, would be different in the Sukemasa and the contemporary dagger. Single-edge analysis is useful to elucidate these characteristics because of its simple calculation method [11].

Figs. 5(a) and 5(b) show a $d_{110}$ and $w_{110}$ distribution around the center of the middle area of the Sukemasa and the contemporary dagger. To obtain high-statistics data for this analysis, BET spectra for a $50 \times 1$ pixel $\left(40 \times 0.8 \mathrm{~mm}^{2}\right)$ area were summed into a single BET spectrum, and stepped at 1 pixel intervals from the edge side to the back side. The $d_{110}$ and $w_{110}$ of the quenched area of the contemporary dagger is obviously larger than the Sukemasa, but the ratio between the hardened and unhardened regions, however, is rather similar; about 1:2. Interestingly, the variation in $d_{110}$ of the back-side region is nearly the same for each sword. This may be because the carbon content of the material and the firing temperature in the manufacturing process were similar for each of these swords [12]. The verification of this last supposition, however, would require the use of additional, destructive testing methods, which is not possible for the Sukemasa.

Figs. 6 (a-c) and Figs. 7 (a-c) show 2D maps of the lattice spacing $d_{110}(\AA)$ and the edge broadening $w_{110}(\AA)$, respectively, of the Sukemasa. As shown in Fig. 5(a), the increase in $d_{110}$ is rather small in the hardened area of the Sukemasa, so that the martensite phase distributions are somewhat vague as shown in Figs. 6 (a-c). However, the martensite phase distributions are clearly seen in Figs. 7 (a-c) and appear roughly correlated with the contrast of Figs. 3 (a-c).

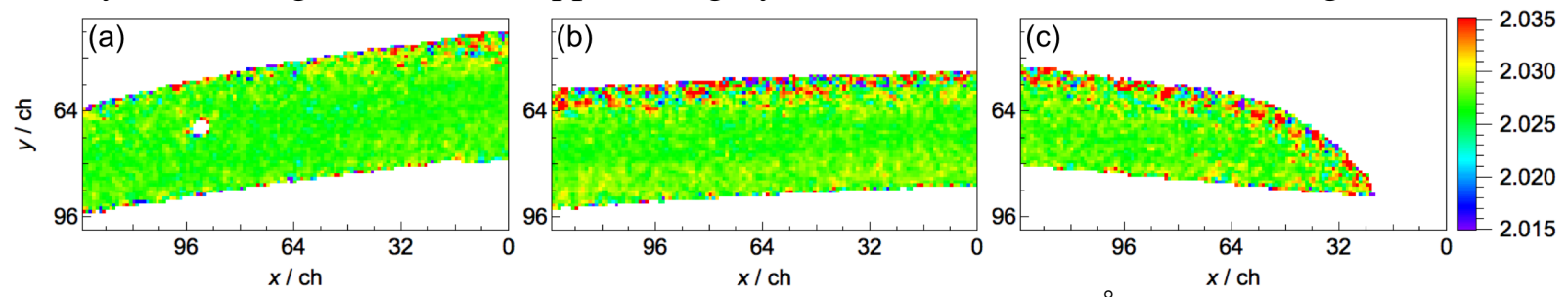

Fig. $62 D$ maps of the lattice (110) plane spacing $d_{110}(\AA)$ of the Sukemasa.

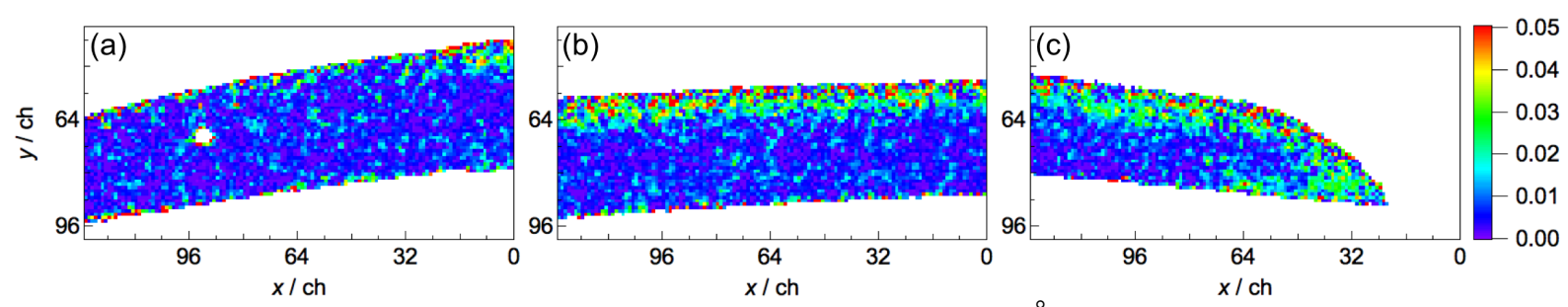

Fig. $72 D$ maps of the edge broadening $w_{110}(\AA)$ of the Sukemasa.

\section{Full-pattern analysis}

In the Japanese sword-making process, the hammering process is repeated several times to remove impurities and ensure uniformity of the blade material. On the other hand, the tang area generally undergoes less processing and different heat treatments compared to the blade area. As shown in Figs. 8 (a-c), the projected density of the sword changes very smoothly, without any wavy/steep fluctuations or voids. In contrast, the tang area exhibits somewhat sudden changes in the crystallite size and preferred orientation parameter as shown in Fig. 9(a) and Fig. 10(a), respectively. 
Figs. 9 (a-c) show maps of the crystallite size of the Sukemasa. Sabine's primary extinction function was used to fit the transmission spectra in the RITS code, where the crystallite size can be evaluated by the extinction parameter, $s$ [13]. The crystallite size in the tip and middle areas is small and almost uniform, in contrast to the tang area, which suggests that the hammering and the quenching processes for the blade area were carried out with great care.

Figs. 10 (a-c) show maps of the preferred-orientation parameter, $r$. The RITS code incorporates a form for the preferred-orientation correction using the March-Dollase (MD) formulation for a cylindrically symmetric distribution [13]. An MD coefficient $r$ provides the degree of the preferred orientation, namely $r=1$ for a random textured sample and $r<1(<\mathrm{hkl}>$ parallel to the incident beam) or $r>1$ ( $<$ hkl $>$ perpendicular to the incident beam) for a textured sample, where $<\mathrm{hkl}>$ represents the preferred-orientation vector. For the present measurement, the preferred orientation $<110>$ for ferrite/martensite gave better fitting results than those using other major orientations like $<100>$ or $<111>$. However, it gave poor fitting results in regions with $r>2$. We tried to fit using other $<$ hkl $>$ close to $<110>$ and found the preferred orientation $<750>$ gave good overall fitting results over the whole range of $r$ found in the sample. The maps indicate that the $<750>$ vector is perpendicular to the incident beam in the present sample setting. The preferred-orientation parameter in most of the blade area, except for the hardened edge, is not so large $(1<r<1.8)$ and the position dependence is small compared with the tang area.
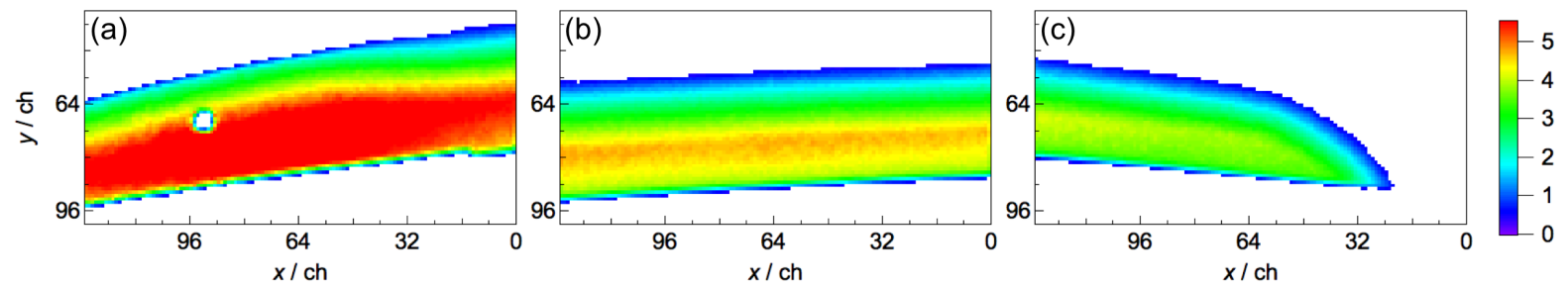

Fig. $82 \mathrm{D}$ maps of the projected density $\rho t\left(10^{22} \mathrm{~cm}^{-2}\right)$ of the Sukemasa. The projected densities for the red area in (a) exceed the maximum scale of the current plots.
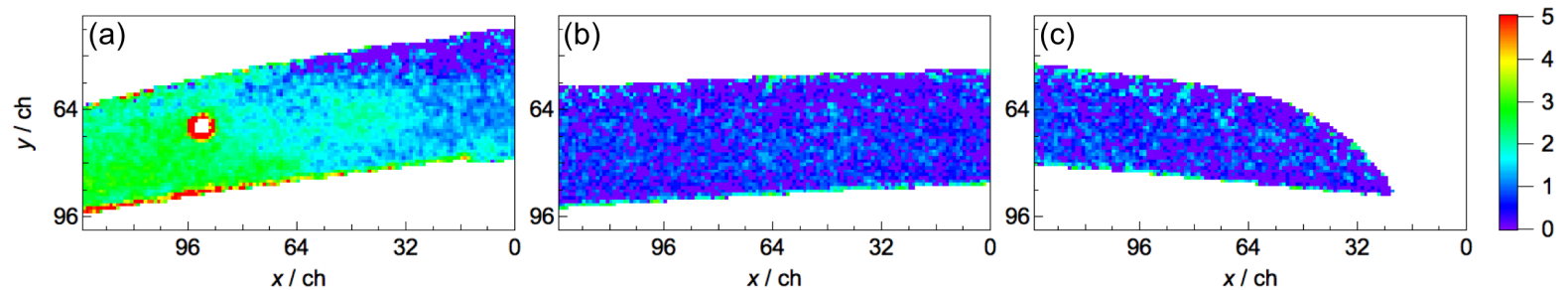

Fig. 9 2D maps of the crystallite size s ( $\mu \mathrm{m})$ of the Sukemasa.
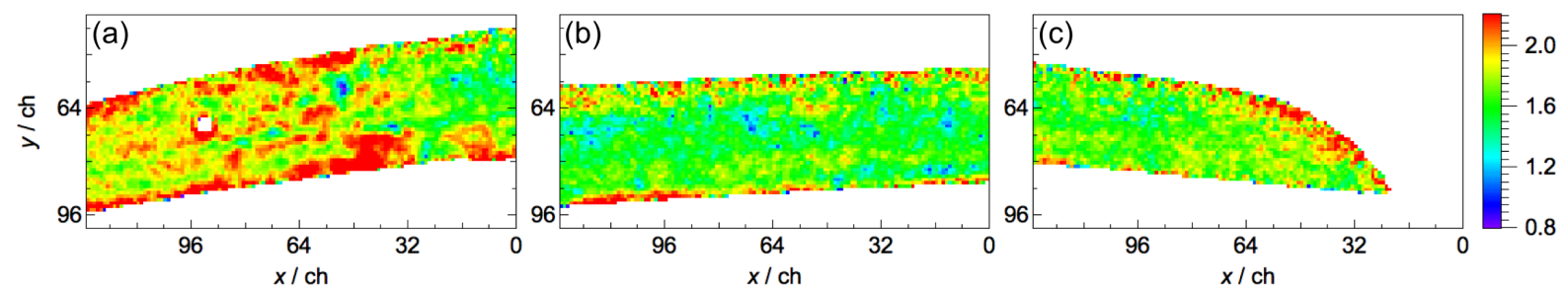

Fig. 10 2D maps of the preferred-orientation parameter $r$ of the Sukemasa. The values of $r$ in the red areas of (a) exceed the maximum scale of the current plots.

\section{Summary}

Crystallographic information of an antique Japanese sword made by Sukemasa was studied using an energy-resolved neutron imaging method. We found the following. 
(1) The wavelength-range contrast images showed increased contrast in the quenching area and its boundary. These contrast images correlate well to the edge-broadening maps rather than the lattice-plane-spacing maps of the Sukemasa.

(2) From an observed increase of the lattice plane spacing and broadening of the edge width, we confirmed that the martensite phase is concentrated in the hardened cutting edge.

(3) Crystallite size seems to be small and almost uniform on the tip and middle areas. On the other hand, the tang area is clearly coarser than the other areas.

(4) The preferred orientation of the tang area seems to be the most anisotropic and the preferred orientation of the hardened edge seems to be somewhat stronger than the rest of the blade.

Together with the evaluation using neutron tomography [14], we will further investigate the detailed information on the micro-structural properties of the Japanese sword Sukemasa.

\section{Acknowledgement}

Neutron experiments at the MLF J-PARC were performed under Proposal Nos. 2017A0099 and 2016B0163. This work partially includes the result of 'Collaborative Important Researches' organized by JAEA, QST and U. Tokyo. The authors would like to thank Japanese swordsmith T. Sasaki for providing test pieces of the dagger sample.

\section{References}

[1] A. Amata, T. Tsuchiko, Tetsu to Nihontō, first ed., Keiyūsha, Tōkyō, 2004 (in Japanese).

[2] T. Saito, Kinzoku ga kataru Nihonshi : senka, nihontō, teppō, first ed., Yoshikawa Kōbunkan, Tōkyō, 2012 (in Japanese).

[3] T. Takahashi, T. Murakami, S. Okada, N. Fujii, Discovering New Aspects in a Japanese Sword, Tetsu-to-Hagane 71 (1985) 108-114 (in Japanese). https://doi.org/10.2355/tetsutohagane1955.71.15_1818

[4] F. Grazzi, L. Bartoli, F. Civita, R. Franci, A. Paradowska, A. Scherillo, M. Zoppi, From Koto age to modern times: Quantitative characterization of Japanese swords with Time of Flight Neutron Diffraction, J. Anal. At. Spectrom. 26 (2011) 1030-1039. https://doi.org/10.1039/c0ja00238k

[5] F. Salvemini, F. Grazzi, S. Peetermans, F. Civita, R. Franci, S. Hartmann, E. Lehmann, M. Zoppi, Quantitative characterization of Japanese ancient swords through energy-resolved neutron imaging, J. Analytical Atomic Spectrometry 27 (2012) 1494-1501. https://doi.org/10.1039/c2ja30035d

[6] Y. Shiota, H. Hasemi, Y. Kiyanagi, Crystallographic analysis of a Japanese sword by using Bragg edge transmission spectroscopy, Phys. Procedia 88 (2017) 128-133. https://doi.org/10.1016/j.phpro.2017.06.017

[7] T. Shinohara, T. Kai, K. Oikawa, M. Segawa, M. Harada, T. Nakatani, M. Ooi, K. Aizawa, H. Sato, T. Kamiyama, H. Yokota, T. Sera, K. Mochiki, Y. Kiyanagi, Final design of the EnergyResolved Neutron Imaging System “RADEN” at J-PARC, J. Phys. Conf. Ser. 746 (2016) 012007. https://doi.org/10.1088/1742-6596/746/1/012007

[8] S. Uno, T. Uchida, M. Sekimoto, T. Murakami, K. Miyama, M. Shoji, E. Nakano, T. Koike, K. Morita, H. Sato, T. Kamiyama, Y. Kiyanagi, Two-dimensional neutron detector with GEM and its applications, Phys. Procedia 26 (2012) 142-152. https://doi.org/10.1016/j.phpro.2012.03.019 
[9] H. Sato, T. Kamiyama, Y. Kiyanagi, A Rietveld-type analysis code for pulsed neutron Bragg-edge transmission imaging and quantitative evaluation of texture and microstructure of a welded $\alpha$-iron plate, Mater. Trans. 52 (2011) 1294-1302.

https://doi.org/10.2320/matertrans.M2010328

[10] B. Dodd, The making of old Japanese swords, J Mech Work Tech 2 (1978) 75-84. https://doi.org/10.1016/0378-3804(78)90016-5

[11] H. Sato, T. Sato, Y. Shiota, T. Kamiyama, A.S. Tremsin, M. Ohnuma, Y. Kiyanagi, Relation between Vickers hardness and Bragg-edge broadening in quenched steel rods observed by pulsed neutron transmission imaging, Mater. Trans. 56 (2015) 1147-1152. https://doi.org/10.2320/matertrans.M2015049

[12] T. Inoue, Tatara and the Japanese sword: the science and technology, Acta Mech 214 (2010) 17-30. https://doi.org/10.1007/s00707-010-0308-7

[13] Y. Kiyanagi, H. Sato, T. Kamiyama, T. Shinohara, A new imaging method using pulsed neutron sources for visualizing structural and dynamical information, J. Phys.: Conf. Ser. 340 (2012) 012010. https://doi.org/10.1088/1742-6596/340/1/012010

[14] Y. Matsumoto, K. Watanabe, K. Ohmae, A. Uritani, Y. Kiyanagi, H. Sato, M. Ohnuma, A.H. Pham, S. Morito, T. Ohba, K. Oikawa, T. Shinohara, T. Kai, S. Harjo, M. Ito, This proceeding. 